\title{
Pneumatic Muscle Actuated Compliant Gripper System
}

\author{
Tudor Deaconescu ${ }^{1}$ and Andrea Deaconescu ${ }^{2}$
}

\begin{abstract}
The paper presents a new constructive solution of a gripper system, actuated by a pneumatic muscle. The proposed variant is based on the utilisation of a linear pneumatic muscle as motion generating element and on motion transmission towards the jaws by means of gears and racks. The main advantages of the proposed gripper system are small weight and low cost, as well as easy mounting on various existing robots. Another important characteristic of this novel gripper system is its compliant behaviour. A compliant gripper system applies forces of adjustable magnitude, thus enabling safe manipulation of objects without causing any deformation.
\end{abstract}

Keywords - compliance, gripper system, pneumatic muscle.

\section{INTRODUCTION}

A SIDE from the more or less evolved mechanisms mimicking human motions developed until the beginning of the $20^{\text {th }}$ century, once with the intensive industrialisation of world economy, with the invention of computing machines and their utilisation for the control of working equipment, the emergence of robots became a natural consequence.

Regarding their aspect, industrial robots look like a human arm. Just like a human arm is attached to the upper part of the body, the industrial robot is attached to a fixed structure known as base. The end-effector of an industrial robot is the gripper system, which typically is not included by the robot's "anatomy".

Of the numerous tasks of the human hand, gripping is the most important one. While the ability to grasp of the limb extremities is also found in animals (for example lobster claws), it is in humans that it has reached its highest functionality. Thus, various combinations of the independent motions of the fingers' phalanges generate the grasping, holding, moving and releasing of objects.

In robotics gripping entails the contact between the endeffector of a robot (the gripper) and a body in view of its manipulation. Grippers are components of robot systems that

Prof. Dr. Eng. Tudor Deaconescu ${ }^{1}$ is with Transilvania University of Brasov, Faculty of Technological Engineering and Industrial Management, Department of Industrial Engineering and Management, Bd. Eroilor 29, Romania, RO-500036 ((corresponding author's phone: 0040-268-477113; fax: 0040-268-477113; e-mail: tdeacon@ unitbv.ro).

Prof. Dr. Eng. Andrea Deaconescu ${ }^{2}$ is with Transilvania University of Brasov, the Faculty of Technological Engineering and Industrial Management, Department of Industrial Engineering and Management, Bd. Eroilor 29, Romania, RO-500036 (phone: 0040-268-477113; fax: 0040-268477113; e-mail: deacon@ unitbv.ro). facilitate the temporary contact with the manipulated objected, ensuring its position and orientation during transport and assembling.

One of the most important aspects of gripping is the stability of the seized object between the gripper jaws (fastness of gripping). Once seized, the object should not move (slip) under the influence of its own weight or of that of inertial forces. The steady position of the object is ensured by the magnitude of the gripping force together with the number of contact points between the jaws and the object.

By the number of seizing areas of the object, gripper systems can have (most frequently) two, three or more fingers (jaws). Studies conducted to date revealed that related to an $100 \%$ gripping ability of a five-finger mechanical hand, this ability is of $99 \%$ in a four-finger mechanical hand, about $90 \%$ three-finger hand, while a hand with two "fingers", namely jaws has a gripping ability of merely $40 \%$ [1].

Many robotic applications like prostheses, medical rehabilitation, and assembling require Variably Stiffness Actuators (VSAs), also known as Adjustable Compliant Actuators (ACAs). These types of actuators offer the possibility of minimising the large forces occurring in collisions (shocks), allow safe interaction with the user and have the capacity of storing and releasing energy into passive elastic elements.

A gripper system driven by a high stiffness actuator is capable of moving a load into a given position with high accuracy, following a pre-set trajectory. Once this position is reached, it is steadily maintained, regardless of the magnitude of the external forces acting upon the actuator. On the other hand, a compliant actuator allows deviations from the position of equilibrium, of amplitudes depending upon the magnitude of the external forces.

Modern gripper systems require the implementation of actuators with a compliant behaviour, such a construction being presented and discussed in this paper.

By the type of actuating energy, gripper systems can be pneumatic, hydraulic, vacuum-based, servo-electric, magnetic, etc. Selection of a certain type of motor depends on a multitude of factors including the weight of the seized object, the required velocity for its gripping, the imposed clamping force, the compliance of the entire system, etc.

Pneumatic actuation is one of the most frequently encountered driving modalities of gripper systems, due to its advantages. These include the simplicity of the control 
diagrams, the possibility of safely overloading the system, easy maintenance, the non-polluting working medium, compliance, etc.

Given the known current state of industrial robot gripper system construction, this paper proposes a novel, light and environment-friendly bionic gripper system. The gripping motions are generated by an original, self-adaptive, bioinspired system deploying a pneumatic muscle as motiongenerating element.

\section{PNEUMATIC Muscle BASED ACTUATION OF GRIPPER SYSTEMS}

Pneumatically actuated gripper systems typically include linear motors that actuate two, three or several jaws (Fig. 1) [2]. Another category of pneumatic gripper systems uses an elastic membrane to seize and fasten a body. These are intrusive type gripper systems that are introduced into the cavity of a body where they change their dimensions (Fig. 2) [2].

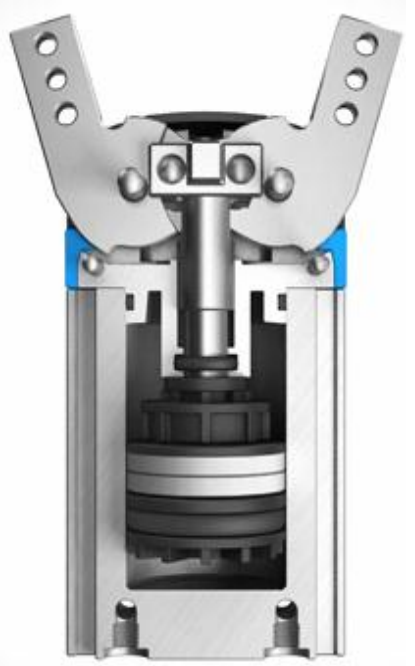

Fig. 1 Actuation by a linear motion

The pneumatic muscle is a relatively new type of linear pneumatic motor. Several constructions of gripper systems based on this motor are known to date, two of these being presented in Fig. 3 [3], [4].

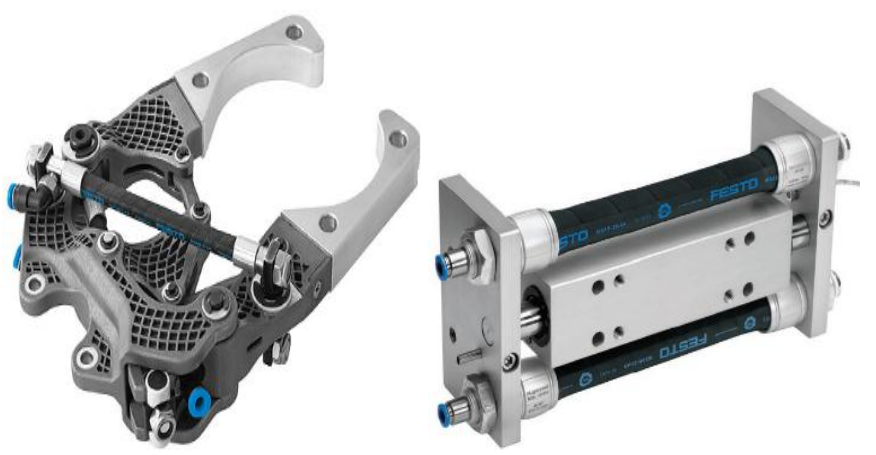

Fig. 3 Pneumatic muscle actuated gripper systems
The first gripper developed by Festo and called Power Gripper started from the way birds use their beaks for picking. The motor is a pneumatic muscle and the gripper construction is based on Watt linkages. The system has a good developed force - to - eigenweight ration, due to the deployment of a light motor (the pneumatic muscle).

The pneumatic muscle is defined as an elastic system built around a contracting membrane, that when fed compressed air increases its diameter und shortens its initial length. The main element of a muscle is a flexible tube covered by a sealed envelope of inelastic fibres in diamond layout that form a tridimensional mesh. When the artificial pneumatic muscle is fed compressed air it is deformed longitudinally, thus generating a pull force.

The behaviour of pneumatic muscles is similar to that of mechanic springs, namely the developed force is at its maximum at the initial moment and then decreases as the deformation of the muscle grows.

The inflation of the muscle under pressure causes it to shorten, while its diameter increases, as can be noticed in Fig. 4. The difference between the no-load length of the muscle ( $p$ $=0$ bar) and its length when fed with air at a pressure different from zero represents the stroke of the muscle.

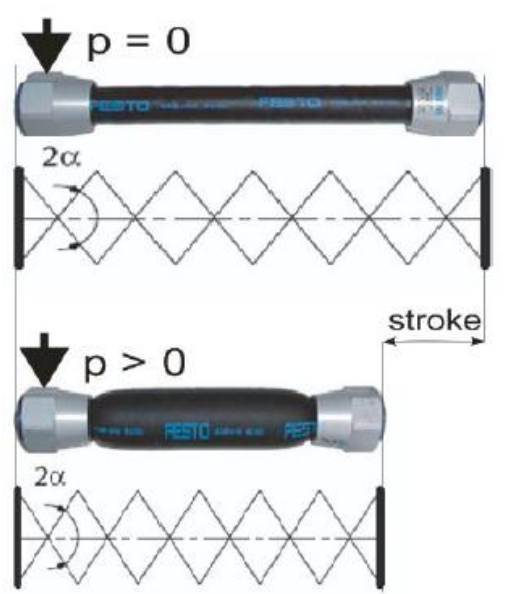

Fig. 4 Working principle of a pneumatic muscle

The pneumatic muscle mimics the functioning of the human muscle fibre (Fig. 5) and benefits from a number of characteristics like shock absorbing capacity and shock resistance, reduced overall size and reduced mass per power unit, and elasticity. These characteristics render pneumatic muscles optimum constructive elements for robotic applications, for orientation mechanisms as well as for grippers.

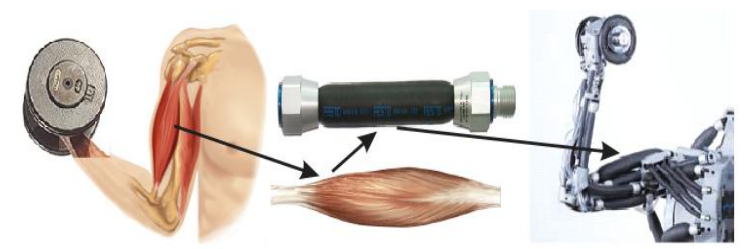

Fig. 5 Pneumatic artificial muscles mimic a biological muscle 


\section{CONSTRUCTION OF THE GRIPPER SYSTEM}

The solution of gripper system proposed in this paper is of non-anthropomorphic type, and lends itself for various robotic applications, of industrial or medical type.

With a pneumatic muscle as the motor element and a gearbased power transmission, Fig. 6 presents a variant of asymmetrical parallel gripper with two mobile jaws.
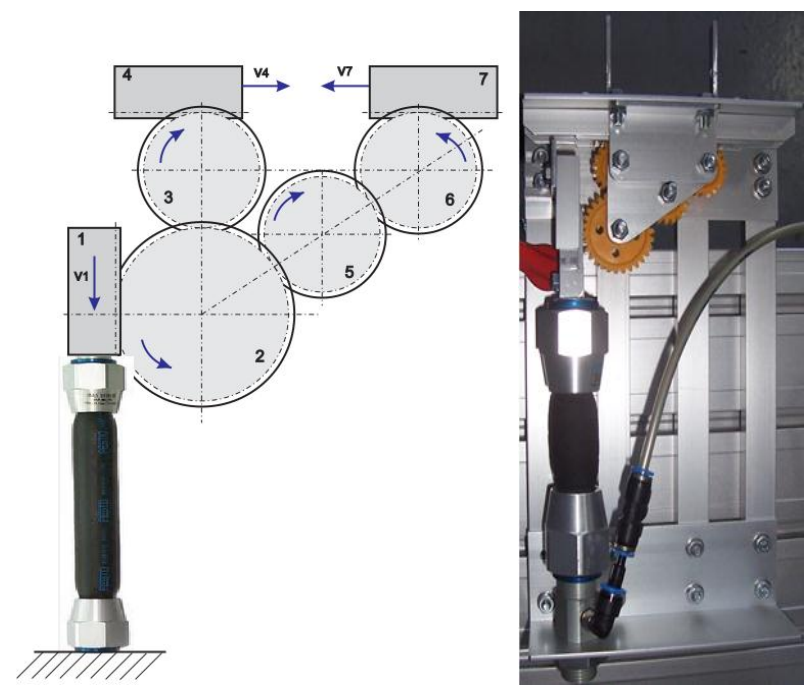

Fig. 6 Asymmetrical parallel gripper with two mobile jaws

The gripper was dimensioned based on the following input data:

- the mass of the manipulated object: $\mathrm{m}=0.7 \mathrm{~kg}$;

- the acceleration of the system: $a=5 \mathrm{~m} / \mathrm{s}^{2}$;

- gravity acceleration: $\mathrm{g}=9.81 \mathrm{~m} / \mathrm{s}^{2}$;

- emergency stop deceleration: $\mathrm{a}_{\mathrm{S}}=10 \mathrm{~m} / \mathrm{s}^{2}$;

- friction coefficient: $\mu=0.2$;

- safety coefficient: $\mathrm{S}=2.5$.

For these input data the maximum force that each of the jaws need to apply onto the seized object is of 83.36 N.

The construction of the gripper system includes the smallest pneumatic muscle manufactured by FESTO AG \& Co: MAS10-45N-AA-MC-O-ER-EG, with an interior diameter of 10 $\mathrm{mm}$ and $45 \mathrm{~mm}$ length of the active part. The main functional characteristics of this muscle are: the maximum admissible pressure is of 8 bar; the axial contraction equals $20 \%$ of the muscle length at rest; the obtainable force is limited to $600 \mathrm{~N}$.

Fig. 7 shows the pneumatic actuation diagram of the muscle. It can be noticed that the compressed air reaches the pneumatic muscle by means of a proportional pressure regulator (MPPES-3-1/4-6-010), controlled by a reference module MPZ-1-24DC-SGH-6-SW (all made by Festo, Germany). By means of rotational potentiometers the reference module can generate up to six different values of the reference voltage, which are transmitted in form of signals to the proportional regulator. If none of these reference values is used, the signal transmitted to the pressure regulator is a voltage adjustable via an external potentiometer.

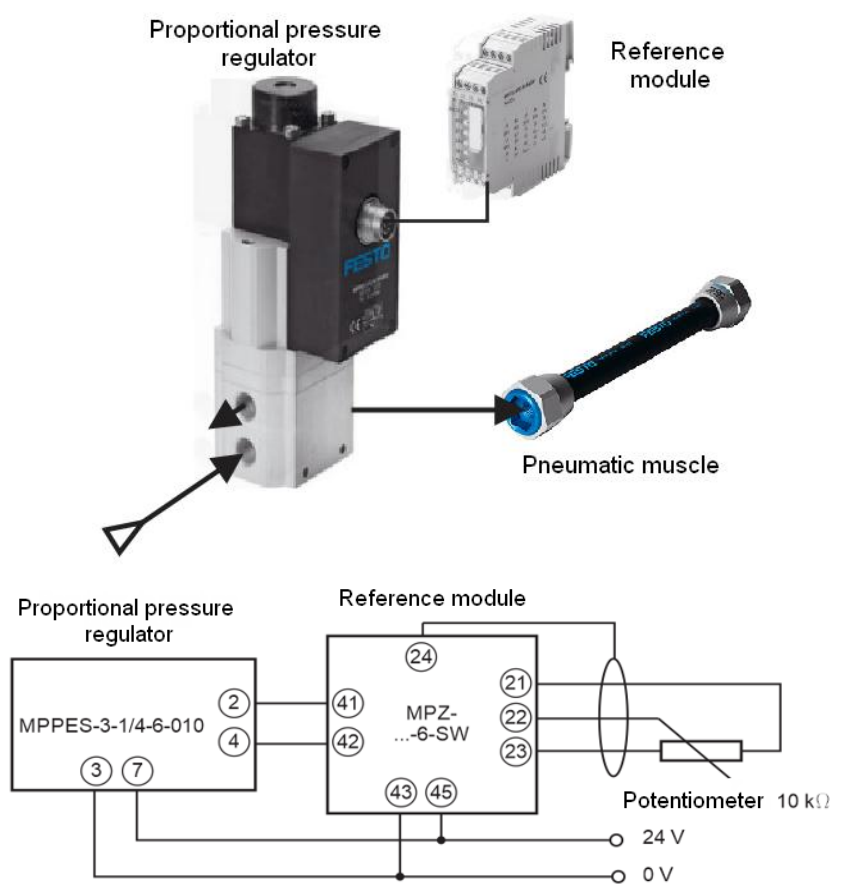

Fig. 7 Actuation diagram of the gripper

\section{DETERMINATION OF THE GRIPPER COMPLIANCE}

Compliance, the inverse of rigidity is one of the most important characteristics required by a gripper system.

A compliant gripper system enables safe manipulation of objects without causing any deformation, by applying forces of adjustable magnitude. Such behaviour can be ensured by using Adjustable Compliant Actuators - ACAs, as is the case of the pneumatic muscle.

Figure 8 presents in the case of a gripper the desired and necessary form of the curve describing the compliance of the entire system.

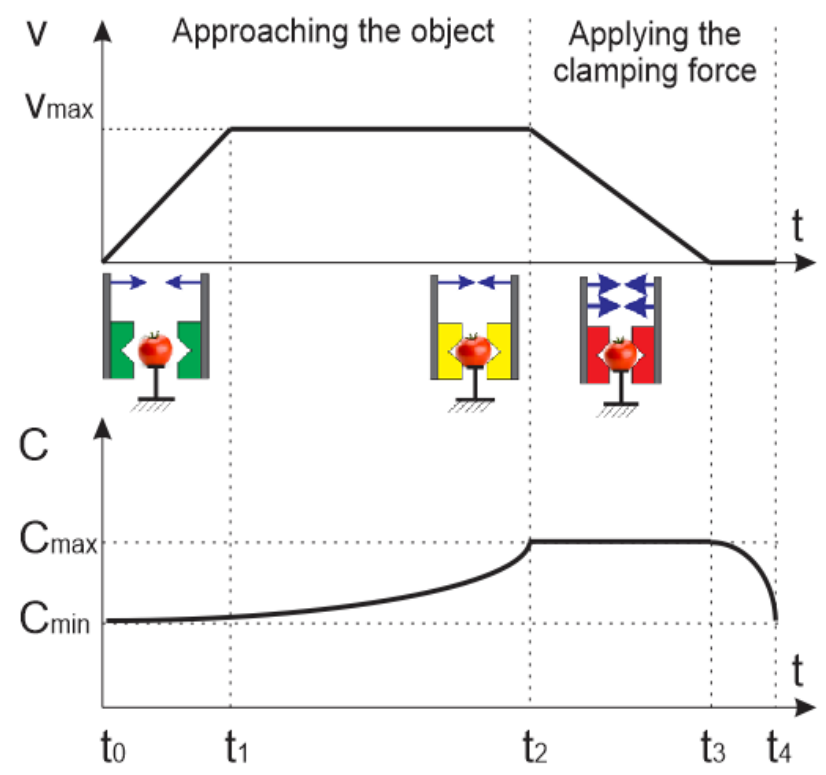

Fig. 8 Variation of the jaw velocity and of the compliance of a gripper versus time 
The two graphs suggest that in the absence of contact between the jaws and the object, at times $t_{0}$ and $t_{2}$, compliance can have smaller values, what allows better positioning accuracy, due to higher rigidity. The curve describing the variation of compliance in this interval needs to be concave, what ensures a more pronounced growth of compliance towards the end of the jaw stroke. Between times $t_{2}$ and $t_{3}$, namely when clamping between the jaws is achieved, the compliance of the gripper system needs to reach its highest values, thus ensuring safe gripping, without the risk of deforming/destroying the seized object.

An adjustable compliant actuator like the pneumatic muscle can adapt its operational behaviour between two limits: thus its behaviour can range from rigid - for high accuracy positioning - to compliant, when the main issue is the safety of the motion [5].

When the dependence between the developed force and the displacement is non-linear, the stiffness of the actuator is not constant. The analysed pneumatic muscle falls into the category of such Variable Stiffness Actuators.

An experimental set-up (Fig. 9) was used in order to determine the variation of the force developed by one jaw of the gripper system depending on the stroke.

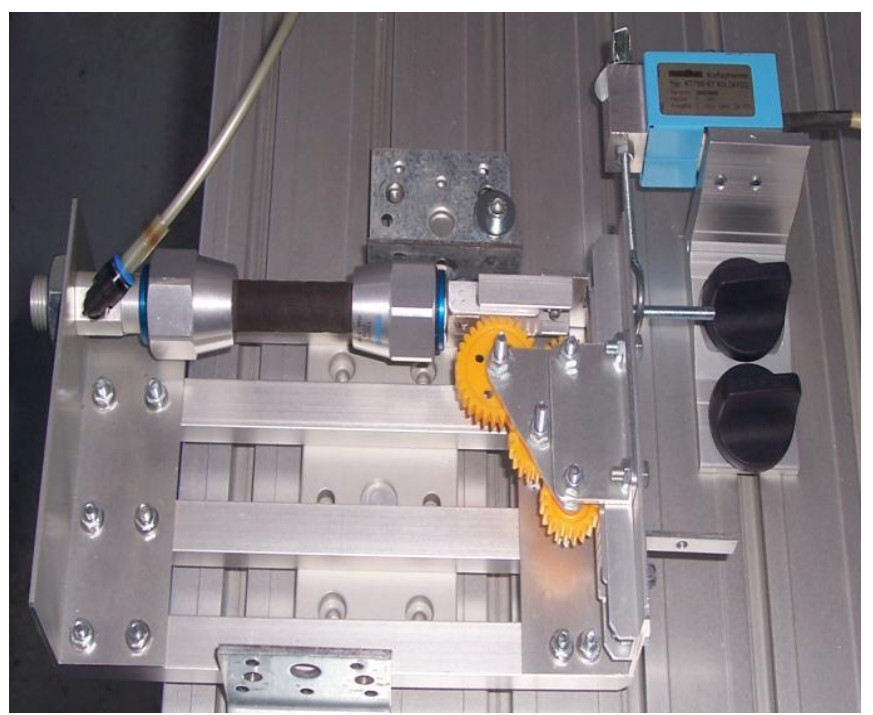

Fig. 9 Measuring the force developed by one jaw

The force transducer is rigidly fixed onto the working table and is attached to one jaw of the gripper system.

In order to measure the forces developed by one jaw, the pneumatic muscle was loaded progressively with compressed air up to 6 bar by means of the proportional pressure regulator.

Fig. 10 presents the variation of the force developed by one jaw versus its stroke, the pressure varying from 0 to 6 bar. It can be observed that at the beginning of the motion the value of the force is at its maximum, and as the jaw progresses towards its limit, the force decreases.

The regression function corresponding to the previous curve is (1) (with a correlation coefficient of $=0.999997$ ):

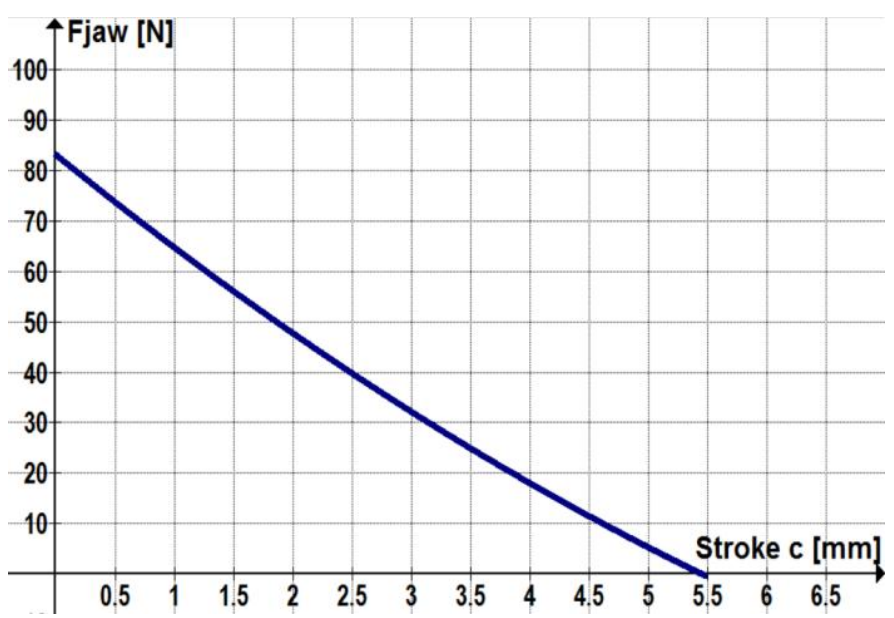

Fig. 10 Variation of the force developed by one jaw vs its stroke

$$
F_{j a w}=83.36-19.146 \cdot c+0.7088 \cdot c^{2}
$$

The rigidity of the analysed system $(k)$ is computed by means of (2). Fig. 11 shows the corresponding graph:

$$
k=-\frac{d F}{d c}=19.146-1.4176 \cdot c
$$

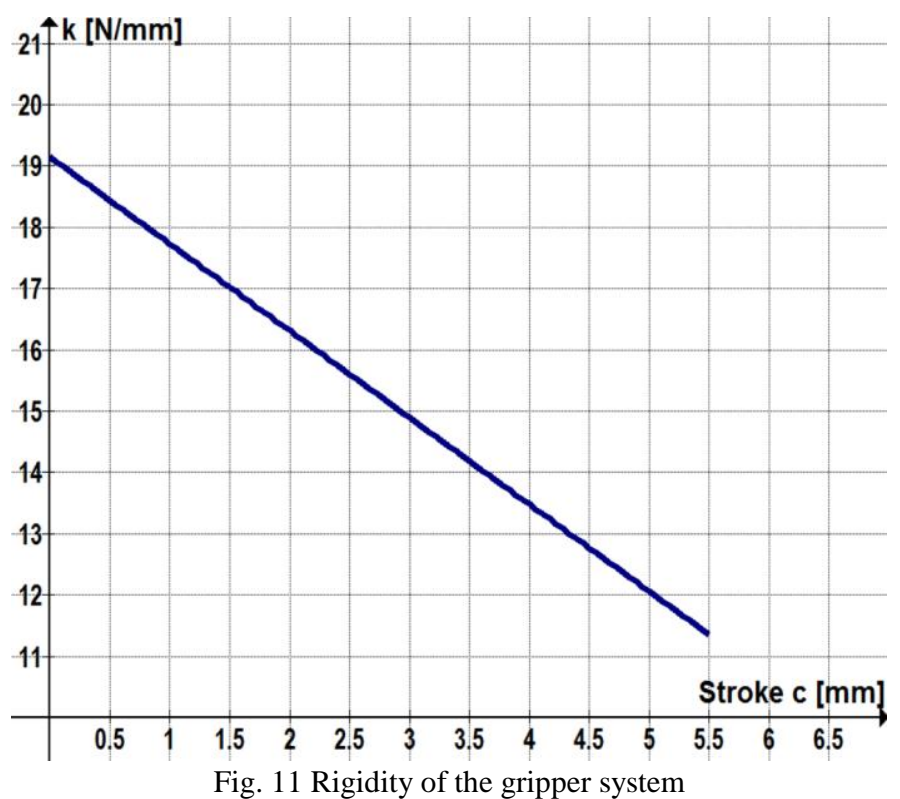

The compliance $C$ of the gripper system is computed as the inverse of rigidity:

$$
C=k^{-1}=\left(-\frac{d F}{d c}\right)^{-1}=\frac{1}{19.146-1.4176 \cdot c}
$$

Fig. 12 shows the evolution of compliance versus the stroke of a jaw. 


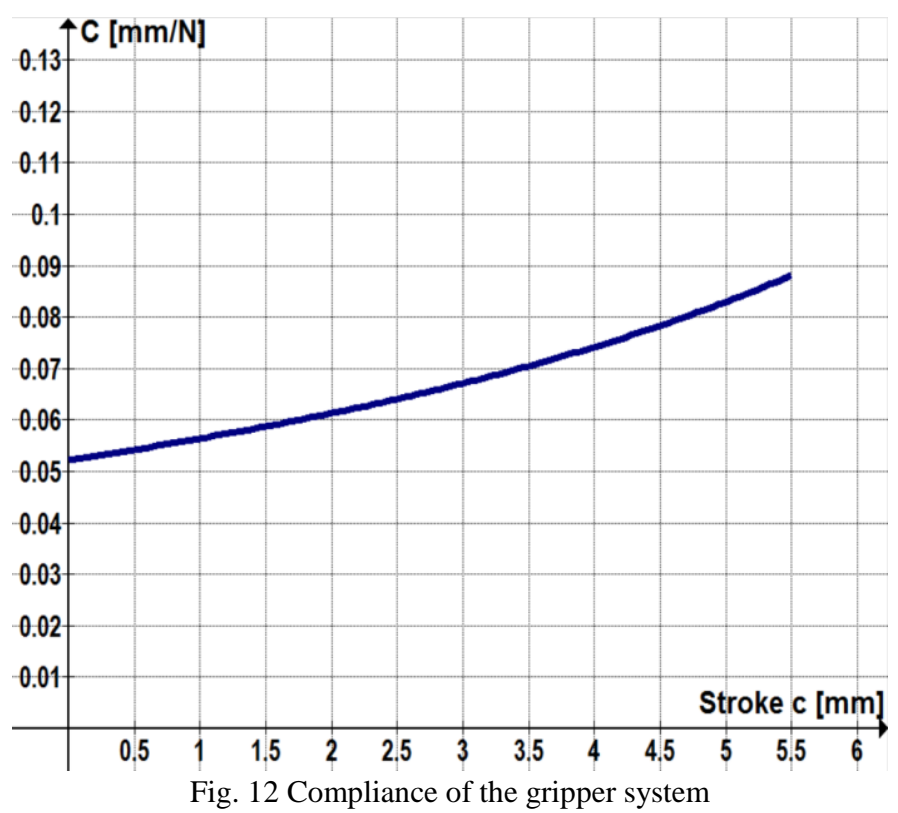

The above graph reveals the concave shape of the compliance curve, what facilitates the accelerated increase of this quantity once the jaw-object contact has been achieved.

Fig. 13 illustrates the analysis of the dependence of rigidity and of compliance, respectively, on the stroke of a jaw and on air pressure.
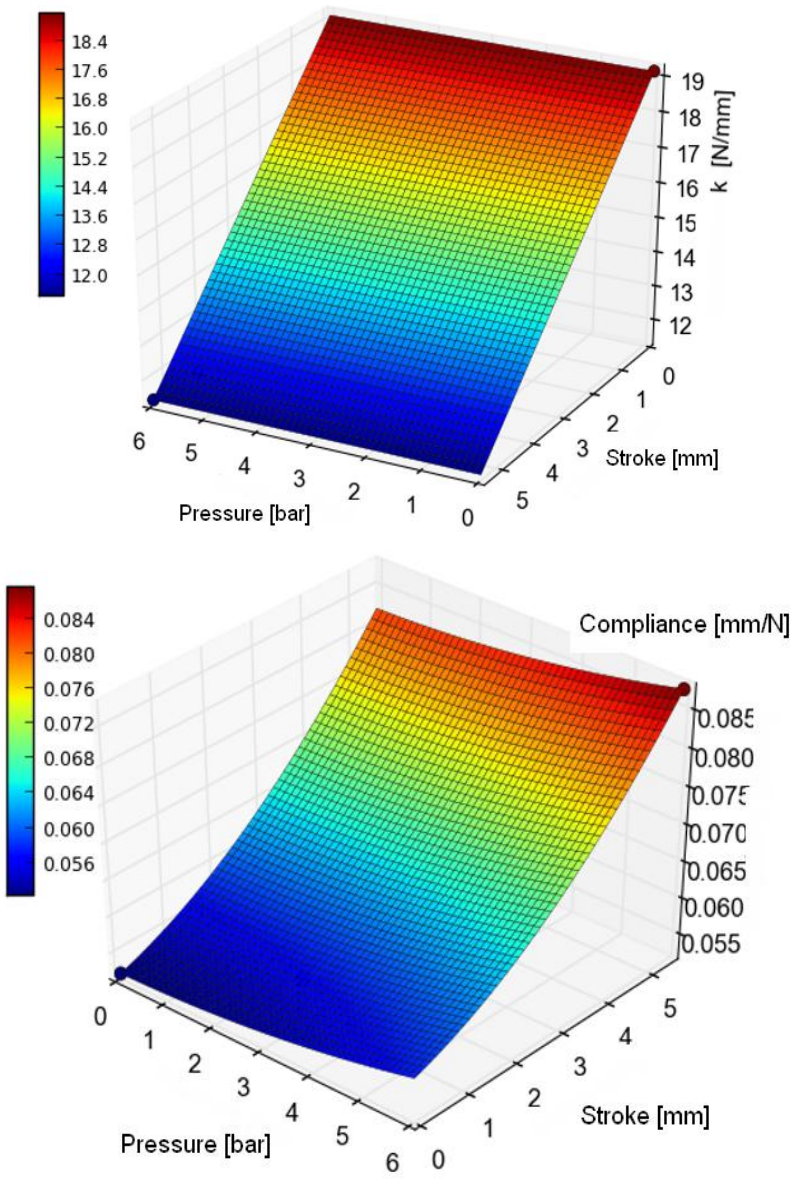

Fig. $13 \mathrm{k}, \mathrm{C}=\mathrm{f}$ (pressure, stroke)
According to the figures above the gripper system is characterised by decreasing rigidity and increasing compliance as the stroke of the jaw progresses and pressure increases. Such evolution of rigidity and compliance causes a longer response time of the system to load variations and, evidently, lower precision. On the other hand however, in systems designed for assembling operations, if for example two components to be mounted are not perfectly aligned, a compliant system, like the one presented in this paper, allows for an adaptive behaviour to the concrete situation, without causing any damage to the components.

The concrete data obtained by measurements allow the defining of intervals of forces and of jaw strokes, respectively, for which the gripper system lends itself. Thus Fig. 14 reveals an area of interest characterised by values of the obtained forces between 50 and $83.33 \mathrm{~N}$, what entails jaw strokes of up to $2 \mathrm{~mm}$.

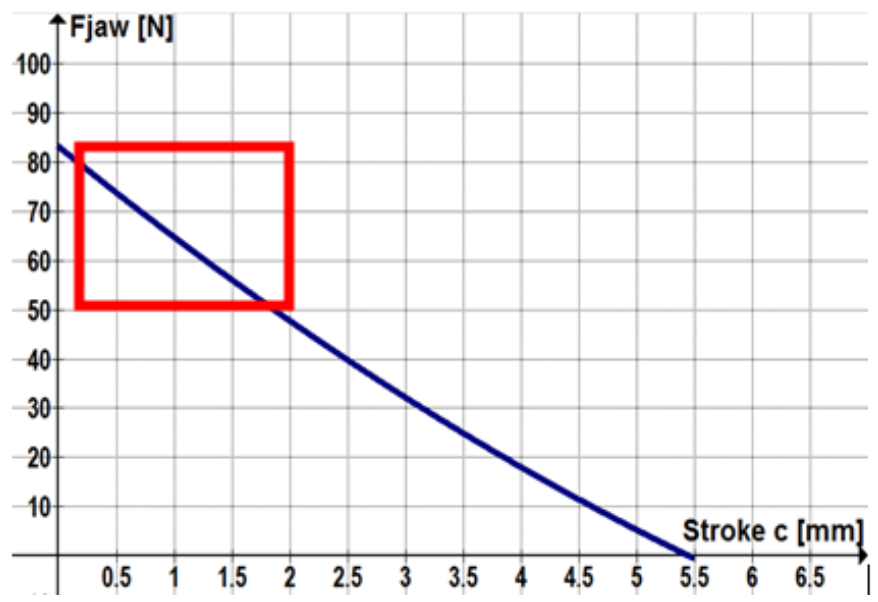

Fig. 14 Highlighting the zone of interest in the deployment of the gripper system

\section{V.CONCLUSION}

The paper presents the construction of a pneumatic muscle actuated novel gripper system, its main characteristic being its compliance. The completed experimental research has revealed the form of the curve that describes the evolution of compliance versus the displacement of the jaw, which allows the seizing with a non-destructive force of the gripped object.

\section{REFERENCES}

[1] C. Stăncescu, Theoretical and Experimental Study of Gripper Systems with Jaws Applicable in Industrial Robots (Studiul teoreticoexperimental al sistemelor de prehensiune си bacuri, си utilizare la roboţi industriali- in Romanian), $\mathrm{PhD}$ Thesis, Transilvania University of Braşov, 2009.

[2] Festo AG \& Co. Germania. Grippers. https://www.festo.com/

[3] Festo. PowerGripper - Research project for the development of new gripper systems. http://www.festo.com/cms/en_corp/12728.htm

[4] Festo. DMSP-...-HGP-SA Muscle Gripper. SA 234717 Product Catalogue.

[5] A. Bicchi and G. Tonietti, "Fast and soft arm tactics: Dealing with the safety-performance trade-off in robot arms design and control", IEEE Robotics and Automation Magazine, vol. 11, no. 2, 2004, pp. 22-33. https://doi.org/10.1109/MRA.2004.1310939 